\title{
MUSICA E MUSICOTERAPIA
}

\author{
Fulvio Šuran \\ Università Juraj Dobrila di Pola \\ Dipartimento di scienze della formazione
}

\begin{abstract}
Riassunto
Perché la musica piace? Molte sono le possibili risposte a tale quesito, nessuna però del tutto soddisfacente. Comunque, di certo nessuno avrà da ridire se si risponde con un "perché fa bene", poiché tale risposta sintetizza un po' tutte le implicazioni della questione, siano esse di ordine biologico, psicologico, sociologico, estetico o etico. In questo senso le relazioni fra educazione generale, educazione musicale e musicoterapia sono assai strette. Ed è nostra intenzione richiamare l'attenzione su di esse, mostrando che la funzione terapeutica non è una questione semplicemente marginale o accessoria dell'educazione, ma qualcosa di ben più rilevante. Per cui è inconcepibile che ancor oggi si possa pensare ad una compiuta educazione, nella quale la musica non sia in onore. Di più, è impensabile un'educazione priva di spirito musicale, inteso come disposizione ad accogliere e ad esprimere, in ben temperata armonia, pensieri ed azioni..
\end{abstract}

Parole chiave: musica, musicoterapia, musica "applicata", ritmo, armonia, melodia, tonalità 
- Belmonte, il giardino della casa di Porzia. Notte.

Entrano LORENZO e GESSICA

\section{Lorenzo}

- Rientriamo, dolcezza, ed aspettiamo in casa il loro arrivo.

Anzi, no, stiamo qui... perché rientrare?

Stefano, amico, va' da quei di casa

e di' che la padrona sta arrivando;

e faccian venir fuori all'aria aperta $i$ vostri musicanti qua all'aperto.

(Esce Stefano)

Come s'adagia soffice la luna col suo riflesso sopra questo poggio.

Noi ci sediamo qui, e lasciamo che l'armonia dei suoni s'insinui dolce dentro i nostri orecchi. La notte con la sua morbida quiete s'addice ad una dolce melodia.

Vieni, Gessica, siedi, guarda l'immensa distesa del cielo come scintilla di patène d'oro: non c'è una stella, per quanto minuscola, che non canti con una voce d'angelo nel suo moto orbitale, e non s'unisca sempre cantando in coro ai cherubini dagli occhi giovani.

E questa musica

sta pur nella nostra anima immortale, anche se noi non possiamo sentirla, finché resta racchiusa in questo involucro nostro d'argilla, rozzo e corruttibile.

\section{(Entrano i MUSICI)}

Avanti, avanti, e risvegliate Diana

con un inno d'amore;

penetrate con le più dolci note nell'orecchio della padrona vostra ed accolgano soavi i vostri suoni il suo ritorno a casa. 


\section{Gessica}

- Non mi riesce mai di stare allegra quando ascolto una dolce melodia.

\section{Lorenzo}

- È perché la tua anima è protesa tutta quanta all'ascolto. Osserva infatti una selvaggia mandria di torelli in foia, o un branco di puledri bradi saltellare sfrenati, e mugghiar alto, come li mena il loro sangue caldo... se appena sentano un suon di tromba, o una musica giunga al loro orecchio, li vedrai arrestarsi tutti insieme, il loro occhio selvaggio convertito in uno sguardo docile e mansueto per il dolce potere della musica. Perciò il poeta immaginò che Orfeo potesse smuovere con la sua lira alberi, pietre, fiumi:

perché nulla è sì duro ed insensibile, e imbevuto di rabbia cui la musica, almeno nell'ascolto, non riesca a mutare la natura.

L'uomo che non ha musica nell'animo né si commuove alle dolci armonie, è nato ai tradimenti, alle rapine, al malaffare, ha foschi e tenebrosi come la notte i moti dello spirito e più neri dell'Erebo gli affetti. Mai fidarsi di uomini siffatti. Ma ascoltiamo la musica.

\section{(da William Shakespeare,} II mercante di Venezia, ATTO V, SCENA I) ${ }^{1}$

\footnotetext{
${ }^{1}$ SHAKESPEARE, W., 2007, The Merchant of Venice, Wordsworth Editions Limited, London, pp. $412-$ 413 (traduzione personale).
} 
Perché piace la musica? Sono stati avanzati molti tentativi di risposta a questa domanda, ma tutti troppo vaghi per essere ritenuti soddisfacenti. In verità la domanda andrebbe meglio precisata come «A chi piace la musica?»; ma la precisazione solleverebbe a sua volta una quantità di problemi di cui non possiamo qui occuparci in dettaglio. Ci limitiamo solamente a dire che la musica sembra piacere agli animali, o almeno ad alcuni di essi ${ }^{2}$. L'obiezione, immediata, che negli animali si può parlare di suoni ma non di musica, non merita molto peso: si tratta della solita, arbitraria demarcazione antropocentrica, tanto decisa e arrogante a priori quanto debole e malsicura a posteriori. Nei casi citati, non sono singoli suoni puri a richiamare l'attenzione, ma veri e propri fraseggi di una o più battute: ed è la forma del fraseggio, quindi l'unita musicale e non semplicemente acustica, che vien ricordata e riprodotta.

Negli insetti (si pensi alle cicale ed ai grilli) o nei batraci l'emissione di suoni è senz'altro più precisa; ma studi attenti hanno rivelato che ogni specie o sottospecie, e perfino ogni singola varietà, produce un suono caratteristico e discriminabile da ogni altro pur simile; lo spettro elettroacustico manifesta anche in questo caso delle sequenze che obbediscono a certe regole di formazione. Biologi ed etologi ci hanno spiegato che queste manifestazioni hanno una precisa funzione di riconoscimento: annunciano una presenza e quindi rivendicano il controllo di un territorio o fanno da richiamo ad un partner. Queste spiegazioni sembrano convincenti al livello più semplice; e forse qualche letterato potrebbe trovare suggestivo che il canto e l'amore siano congiunti fin dall'inizio. Ad ogni modo, come in molte altre funzioni c'è qualcosa in più, che va oltre la prima e fondamentale spiegazione; si potrebbe dire che, a un certo punto, il canto prende la mano al cantore, si sviluppa per conto suo. L'autonomizzarsi della funzione, oltre a svolgerne la intrinseca possibilità, prelude al suo esprimersi libero, come gioco. Nel canto dell'usignolo, a parte le funzioni etologiche, è difficile non sentire l'insistenza dell'auto-ascolto, qualcosa che, a modo nostro, chiameremmo un compiacimento del cantore: che fra l'altro è alla base delle variazioni controllatissime, eppur creative, della sua espressione musicale assai complessa. Ma limitiamoci pure al suono.

\footnotetext{
2 Prescindendo dai serpenti, relativamente ai quali sussistono dubbi, pare piaccia ai delfini, alle balene, agli elefanti, alle scimmie; ma, soprattutto, il mondo canoro per eccellenza è quello degli uccelli, che dispiegano una varietà sorprendente di combinazioni musicali.
} 
Per quanto ne sappiamo, le civiltà che si sono sviluppate prima o al di fuori di ogni altra tradizione scritta hanno avuto comunque una ricca tradizione orale, alla quale si è sempre associato il canto, spesso unito alla danza, con il supporto di semplici strumenti musicali. Perfino le maschere di paglia o di legno intagliato (e quindi un'altra forma d'arte che è stata alle origini della scultura) hanno avuto impieghi principalmente festivi o rituali collegati a rappresentazioni mimate e danzate con accompagnamento di battimani, percussioni, musiche e canti. Quello che tra la fine del secolo scorso e l'inizio di questo è stato chiamato folklore sarebbe inconcepibile senza musica.

Le dimensioni principali della musica, senza pretendere di esaurirne gli aspetti, sono:
a) il ritmo,
b) l'armonia,
c) la melodia.

a) II ritmo è senza dubbio la componente fondamentale, e già da solo, attraverso le percussioni, costituisce un'espressione musicale autonoma; il ritmo è una quantificazione periodica di una emissione di energia, ed è pertanto collegato in misura determinante alla percezione del tempo e dei suoi intervalli. Poiché l'orologio fondamentale è per noi quello biologico, il carattere ciclico e ricorrente di alcuni fenomeni ${ }^{3}$ è probabilmente alla base della nostra sensibilità al ritmo. Un ritmo lento può esprimere, ma anche accompagnare e trascinare con sé stati fisiologici più distesi e tranquilli di un ritmo veloce, o di uno interrotto e saltellante. Se un ritmo acustico fa da commento alla danza, che è un ritmo cinetico, l'associazione può essere perfetta, e i suoi effetti psicosomatici possono essere imponenti: dal coinvolgimento moderato di un minuetto, a quello più appassionato di un flamenco, fino allo stato di trance indotto dalla danza vorticosa dei dervisci.

Il ritmo è anche la prima forma musicale accessibile ai bambini; non occorre rammentare l'effetto soporifero delle ninne-nanne, o quello allegro e giocoso dei girotondi. Proponendo strumenti occasionali, i bambini possono realizzare un piccolo concerto di percussioni, agendo non solo da esecutori ma altresì da compositori.

\footnotetext{
${ }^{3}$ Dal battito cardiaco alle onde elettriche EEG associate al lavoro cerebrale, per non dire di molti altri.
} 
b) Nella sua forma sviluppata l'armonia regola le consonanze e gli accordi, ma nella sua base strutturale la sua radice si trova nell'esistenza di scale musicali, cioè di successioni di suoni disposte secondo certi rapporti. È interessante osservare che un suono puro, una nota, può entrare in diverse scale con ruoli diversi. In tal senso le note valgono quindi non solo per se stesse, ma per i rapporti che intrattengono col proprio contesto.

Molte musiche infantili e anche molti canti e danze popolari sono espresse in una scala pentatonica ${ }^{4}$; il patrimonio della musica occidentale, nella classica scala eptatonica ${ }^{5}$; alcune composizioni moderne, nella scala dodecafonica ${ }^{6}$.

L'insieme delle relazioni che sussistono fra le note di una scala costituisce la tonalità.

Come è noto, si può far slittare una musica all'insù o all'ingiù lungo una scala, purché siano mantenuti i rapporti fra le note che la costituiscono. Questo costringe a introdurre dei semitoni ${ }^{7}$, perché i rapporti non cambino partendo da un diverso gradino ${ }^{8}$, ma tutti sanno che l'operazione è soggetta ad alcuni vincoli, e che non produce il medesimo effetto.

Così, per esempio, tradizionalmente alcune tonalità si ritengono più adatte a esprimere sentimenti magnanimi ed esaltanti ${ }^{9}$; altre, sentimenti elegiaci e tristi ${ }^{10}$.

Si discute se l'essere stonati o intonati sia nell'ordine delle doti naturali, cioè una proprietà innata o acquisita. È molto probabile che la predisposizione sia innata, ma certo l'esercizio guidato può far molto per migliorarla. D'altra parte, è impossibile suonare a due mani e con più dita ${ }^{11}$, senza possedere qualche senso dell'armonia. I bambini sembrano abbastanza disponibili a tale attività, specialmente se in età precoce ${ }^{12}$. Non bisogna comunque confondere la perizia tecnica, che richiede lunga fatica, con la disposizione naturale. I vertici dell'armonia, raggiunti storicamente da grandi maestri della musica strumentale, come J. S. Bach, o corale, come P. L. da Palestrina, rappresentano certamente la

\footnotetext{
${ }^{4}$ A cinque gradini.

${ }^{5}$ Di sette note; due toni, un semitono, tre toni, un semitono.

${ }^{6}$ A dodici gradini, tutti di un semitono.

${ }^{7}$ Diesis o bemolle.

${ }^{8} \mathrm{O}$ semi-gradino.

${ }^{9}$ Per esempio quella in si bemolle maggiore.

${ }^{10}$ Per esempio quella in do minore.

${ }^{11}$ Ovvero producendo su uno strumento più note contemporaneamente.

${ }^{12}$ Pur senza forzare l'argomento valendosi di eccezioni come il piccolo W. A. Mozart.
} 
migliore approssimazione alla metafora della musica come armonia delle sfere ${ }^{13} \mathrm{e}$ come metafisica inconsapevole ${ }^{14}$. Ma anche in forme più modeste, non c'è musica da concerto od operistica, duetto o trio o quartetto che ne possa ormai prescindere.

c) La melodia o, etimologicamente, il canto è la frase musicale. Le note isolate possono anche esser piacevoli ma non hanno valore musicale ${ }^{15}$; poste in scala, ne hanno; suonate assieme, producono consonanze o dissonanze; ma solo in quanto sono poste in successione, con valori e durate differenti, in modo da costruire delle frasi, acquistano pienezza di espressione e capacità di comunicazione ${ }^{16}$.

Si può teorizzare (ed è questa la mia convinzione) che sia stato il caso, giocando sull'inesauribile combinatoria dei suoni producibili, a produrre frasi melodiche innumerevoli, alcune delle quali hanno avuto la meglio. Con questo, il problema è spostato dall'origine alla scelta: ma è veramente difficile dire perché una melodia sia preferibile ad un'altra.

La distinzione fatta fra le tre dimensioni accennate è - non occorre insistervi - del tutto relativa. L'invenzione musicale produce infatti un intero nel quale ritmo, armonia, melodia sono reciprocamente integrati in funzione reciproca.

Ed è questo intero ad agire sulla nostra sensibilità e sulla nostra ragione, come un persuasore occulto penetrante e assiduo. Gli antichi favoleggiavano di Orfeo che, con la propria musica, muoveva macigni, e Plutarco rammentava quant'erano terribili le truppe degli opliti spartani che marciavano ordinati al combattimento al suono del flauto ${ }^{17}$.

Qualsiasi rito religioso o civile perderebbe i due terzi della sua efficacia coinvolgente se non fosse scandito dalla musica: che salda, fonde, muove le folle e fa crollare le mura (come quelle di Gerico).

\footnotetext{
${ }^{13}$ Qui si pensa alla cosmogonia numerica di Pitagora.

${ }^{14}$ Del filosofo tedesco A. Schopenhauer.

${ }^{15} \mathrm{E}$ qui dovrebbe entrare in gioco il discorso sull' effetto del timbro, che qui abbiamo escluso.

${ }^{16}$ Alcuni uccelli esprimono brevi frasi di 2-3 note, altri melodie assai complesse. Nell'uomo è probabile che le prime melodie abbiano preso spunto da frasi naturali esprimenti gioia, tristezza, minaccia: ma per questa via sarebbe assai difficile procedere oltre i primi passi, come per la via onomatopeica per spiegare le origini del linguaggio.

${ }^{17}$ Non diversamente calavano al rullo dei tamburi nelle nebbie di Austerlitz gli eserciti dei tre imperatori, o combattevano nelle guerre coloniali gli high-landers scozzesi.
} 
C'è indubbiamente qualche corrispondenza recondita con i ritmi arcaici, con l'eccitazione e il rilassamento, con le costellazioni associative e umorali, che fa della musica un tipo di comunicazione sui generis: certo non denotativa e referenziale, non analitica e segmentabile, ma contagiosa e altamente partecipativa. Una comunicazione da mano sinistra ed emisfero destro.

Per questo, le tecniche orientali di concentrazione e meditazione, se al loro culmine esigono il silenzio, possono tuttavia utilmente avvalersi della musica nella fase preparatoria e introduttiva.

Per questo, esiste una musicoterapia che esercita una preziosa efficacia sui soggetti che soffrono di varie disarmonie nelle coordinazioni senso-motorie, o che hanno difficoltà di comunicazione verbale, o turbe psichiche di varia natura; si direbbe che in questi casi la musica agisca come un regolatore: rafforza ed allea altri regolatori e normalizza i processi vitali e gli scambi con l'ambiente.

La risposta alla nostra domanda iniziale "perché piace la musica?" potrebbe a questo punto essere "perché fa bene» - in tutti gli aspetti del vivere umano. Sarebbe tuttavia una risposta banale, se non fossimo capaci di leggere entro quel far bene tutte le considerazioni fin qui fatte; di ordine biologico, psicologico, sociologico, estetico ed etico. La natura obbedisce ovunque a leggi d'ordine ${ }^{18}$, e la musica è una delle più potenti espressioni delle leggi d'ordine nella loro processualità temporale ${ }^{19}$. Piena corrispondenza fra le varie forme d'ordine che si riflette poi, di gradino in gradino, su tutti i piani dell'essere, e può essere riscoperta in ognuno di essi; la forma acustica, se possiamo usare un bisticcio di parole, vale come l'eco di un'armonia più profonda.

\footnotetext{
${ }^{18}$ Anche il caso non può che scegliere uno fra gli ordini possibili.

${ }^{19}$ Le forme infinite dei radiolari e delle diatomee, le spirali delle conchiglie avvolte con innumerevoli varianti potrebbero esser chiamate a confronto: ma in esse prevale il momento della forma definitiva, statica, come nei cristalli: mentre nella musica l'ordine arcano, la morfogenesi eterna si dispiega nel suo farsi attivo, come legge ascendente e creativa. II filosofo speculativo non esita perciò a vedere nel Bello la controparte del Bene, la sua misurabile manifestazione.
} 


\section{Non c'è musica «applicata»}

Le relazioni fra educazione generale, educazione musicale, musicoterapia sono assai strette.

È qui importante richiamarle, perché non avvenga di considerare la funzione terapeutica come semplicemente marginale o accessoria, o come una applicazione della musica. Chi così pensa, ha un'opinione tutto sommato non elevata della musica, considerandola alla stregua di un coadiuvante distensivo e nulla più.

Bisogna dire che la musica non è mai stata in se stessa qualcosa di applicato: o meglio, se talvolta suo malgrado lo è stata, ciò è stato dovuto ad una sorta di offensiva dimenticanza della sua natura intrinseca: in tali casi è stata infatti svilita ad un compito servile: come quando era (e, frequentemente, ancor oggi è) chiamata a fornire nient'altro che uno "sfondo sonoro" a banchetti o spettacoli, come un addobbo superfluo. Un pubblico elegante si distraeva e chiacchierava non porgendo attenzione alla musica ${ }^{20}$. C'è da dire che ai nostri giorni la cosa è diventata cronica: c'è gente che tiene accesa tutto il giorno la radio o la televisione pur senza porgervi ascolto. Si tratta di un bisogno analogo a quello della chiacchiera fitta e insistente su non importa quale contenuto, un generico riempimento del vuoto, che denuncia per altro il vuoto stesso delle persone in questione ${ }^{21}$.

\section{L'educazione al silenzio}

Si potrebbe dire, paradossalmente, che per tutte queste persone il primo passo verso l'educazione alla musica dovrebbe essere l'educazione al silenzio.

La parola e la musica hanno bisogno di spiccare sul silenzio.

Quando noi siamo attenti a un discorso o a una musica, infatti, dobbiamo imporre in qualche maniera un silenzio - almeno psicologico- a tutto il resto, come se non ci fosse.

\footnotetext{
${ }^{20}$ Era la stessa funzione assegnata alla pittura resa semplice decorazione o tappezzeria.

${ }^{21}$ Il bisogno diventa compulsivo fino a dilagare al di là dei limiti della normalità nella pratica dell'ascolto ininterrotto, reso oggi possibile dai vari Lettori MP3 o FM Digital Radio: strumenti che potrebbero essere utili per l'ascolto individuale discreto, se non fossero invece diventati invasivi in situazioni quanto meno improprie. È quindi motivo di perplessità constatare fra l'altro, come si è fatto in recenti indagini, che mentre il $70 \%$ dei giovani acquista dei $C D$, meno del $20 \%$ è anche disposto a far davvero musica attivamente in qualche forma reale.
} 
L'attenzione è selettiva, filtra il segnale desiderato ed elimina il disturbo. Ovviamente, è ancora meglio se il disturbo è eliminato non solo in maniera psicologica, ma fisica. Non occorre rammentare, del resto, che il silenzio è necessario non solo come condizione d'ascolto al contorno, ma anche come condizione strutturale interna allo stesso discorso musicale. Ma, ancora di più, sono gli intervalli che producono nei confronti di suoni di diversa altezza i rapporti da cui nascono la melodia e l'armonia.

Tuttavia, la creazione di un ambiente sonoro come dato permanente della nostra civiltà sembra ormai un fatto acquisito. Migliore delle precedenti applicazioni ma ancora marginale, è per esempio la diffusa pratica di creare un sottofondo musicale in certi ambienti ${ }^{22}$ facilitato oggi dalla filodiffusione.

In questi casi la musica concilia uno stato d'animo e favorisce il rilassamento; siamo sulla soglia di un impiego terapeutico, ma in senso molto lato e generico. Rimane fondamentale in tutti questi casi la differenza fra il puro e semplice "udire» e l'ascoltare con attenzione.

\section{La musica tra il labile e il cosmico}

La musica è ben altro: essa fluisce, dal soggetto che la crea, in una con il gesto e la parola: è espressione piena al pari di questi ultimi e, quando nasce spontaneamente, è parte indissolubile del messaggio totale. Già il gesto pone la base per il ritmo, e la parola non prescinde dalla intonazione, che è soprasegmentale e gestaltica, quindi globale e inviluppante ${ }^{23}$. Del resto, già chi zufola o canticchia, sentendone quasi il bisogno naturale, sa che la sua espressione nasce intera così, e se ne fosse impedito resterebbe sorpreso e contrariato.

Una spontanea letizia non si potrebbe esprimere con parole in codice, come un freddo telegramma.

D'altra parte, neppure un dolore può prescindere da una modulazione di tipo musicale: e lamentazioni e pianti funebri hanno sempre avuto cadenze che si sono facilmente ritualizzate.

Quando si passa da queste manifestazioni primitive ad espressioni più complesse, il genio creativo popolare fissa motivi di danze e canzoni che si tramandano per secoli, fino a costituire un repertorio stabile. Più oltre ancora, si sale ai vertici dell'espressione musicale con la musica corale, l'innologia sacra, la

\footnotetext{
${ }^{22}$ Come ad esempio alberghi, circoli, cliniche, supermercati, sale d'attesa.

${ }^{23}$ Esistono lingue cantate, come il cinese, che non potrebbero essere altrimenti capite.
} 
musica strumentale da camera e sinfonica, il dramma in musica. Ma ancora ai giorni nostri non c'è genere di comunicazione o di spettacolo, come non c'è nuova tecnologia della comunicazione che presto o tardi non sia costretta a fare i conti con la musica.

Fra lo spontaneo motivetto sussurrato a fior di labbra o semplicemente immaginato e la grande musica corre certo una grossa differenza: più di grado, però, che di natura essenziale. In quanto la cosa veramente importante è l'identità intuizione-espressione: che, nel caso nostro, equivale a dire che la musica deve essere intimamente sentita ${ }^{24}$.

Chi non sente la voce del cuore nelle note del pianoforte che gocciolano piano invitando alla mestizia, o che travolgono in una polacca che esorta con foga allo slancio verso alte imprese?

Come il leggere un testo e capirlo vuol dire saperlo ricostruire in sé, così I'ascoltare musica con intendimento vuol dire saperla in qualche modo riprodurre ${ }^{25}$. Perciò gode appieno della musica chi sa fare musica.

Il vero ascolto non può mai esser passivo, senza ricadere in qualche forma di abuso che tratti la musica come un qualcosa di superfluo e aggiuntivo.

\section{Il trapasso dai suoni alla musica}

Il problema dell'educazione musicale comincia dunque dal riconoscimento della natura propria del linguaggio musicale: che è linguaggio non referenziale ${ }^{26}$, ma è linguaggio a pieno titolo, in quanto si serve di un supporto fisico per trasmettere contenuti psichici. È quindi mezzo di comunicazione e tramite di pensieri e di sentimenti, anche se in forme più globali e sintetiche di quelle proprie della lingua parlata o scritta.

Al di sotto di questo livello, non c'è più musica, anche se ci può essere una realtà fonico/acustica non priva di interesse: rumori e suoni, che pure svolgono delle funzioni nel nostro complesso rapporto con l'ambiente, e costituiscono una

\footnotetext{
${ }^{24}$ Da questo punto di vista un semplice motivetto può essere sentito, da chi è privo di talento e di cultura musicale, di più, e non di meno della grande musica da concerto Prova ne sia il fatto che parecchi si annoiano ai concerti di musica classica; si annoiano perché non sono capaci di ricreare in sé stessi la gioia della genitura spirituale di quella musica, che resta inesorabilmente al di sopra delle loro possibilità.

${ }^{25}$ Certo, se leggendo Platone o Dante noi ci identifichiamo per qualche istante con i loro pensieri, e tanto più li gustiamo intimamente quanto più vorremmo poterli esprimere come parola nostra, altrettanto ascoltando Beethoven o Chopin ci eleviamo nella misura in cui, guidati da loro, facciamo risuonare in noi la stessa eco.

${ }^{26}$ In quanto non è linguaggio da vocabolario.
} 
sorta di materiale acustico, destinato tuttavia ad essere dominato e trasformato per convertirsi in musica propriament detta.

Da questo primo riconoscimento discendono subito alcuni tratti della educazione musicale naturale: che deve prendere avvio dall'ascolto e produzione di sequenze ritmiche e tonali semplici e spontanee, per passare gradualmente a dominare sempre più la materia sonora. Il passaggio dai rumori e suoni alla musica si ha infatti con la sequenzializzazione misurata da rigorose regole, in forme dapprima incoscienti e poi via via più consapevoli.

L'unità musicale elementare non è il suono di una nota pura, bensì la frase, ossia la totalità, piccola o grande - vi sono infatti anche totalità semplici, all'interno di altre totalità più complesse- che condiziona il ruolo e la funzione delle sue parti, assegnando loro un significato ed un valore che non possono in alcun modo prescindere da quell'insieme. Elementi singoli avulsi da quell'insieme, se inseriti in altri insiemi, diverrebbero a loro volta altri, e pertanto irriconoscibili ${ }^{27}$.

\section{C'è metodo e metodo}

Si disputa oggi sui metodi migliori per insegnare musica a tutti.

È evidente che la preparazione tecnico-artistica dei musicisti costituisce un serio problema, che qui non ci riguarda se non di striscio, in quanto ci si chiede se coloro che devono insegnare la musica a tutti debbano a loro volta essere musicisti oppure no.

Abbiamo seguito e ammirato la bravura, la sapienza didattica e -perché no? - l'ispirazione di maestri come Willems, Dalcroze, Orff, Kodàly, Ward, la Bassi ed altri.

Senza entrar nel merito della mia perizia musicale, posso dire di aver visto e ascoltato bambini felici perché il loro maestro li faceva cantare e in molti casi accompagnare il canto con qualche facile strumento.

Ritengo che, se sul piano strategico non è possibile indicare un metodo da favorire a priori in modo esclusivo, sul piano tattico sia d'altra parte necessario, una volta che una qualche scelta sia stata operata, rimanere fermi nell'applicarla. Anche perché ogni didattica della musica ha molte possibilità e dimensioni, nelle quali convivono senza disturbarsi canali diversi, ognuno con le sue regole proprie.

\footnotetext{
${ }^{27}$ Una stessa nota assume significato diverso da una tonalità all'altra; ma ha anche significato diverso entro la stessa tonalità a seconda della frase musicale in cui è inserita. Il timbro e la dinamica aggiungono altre distinzioni specifiche.
} 
D’altra parte il riferirsi ai suoni (come ai colori) non vuol dire ridursi ad un livello fisiologico di pure sensazioni irrelate, come qualcuno potrebbe erroneamente intendere, ma piuttosto esser consapevoli e capaci di dominare i mezzi costruttivi dell'arte: così che essa non si limiti al momento dell'ascoltare (o, nel caso affine, del guardare) ma includa anche il momento del fare ${ }^{28}$.

\section{La musicoterapia nell'intreccio educativo}

La musicoterapia, benché solo da poco entrata nella fase scientifica, ha in verità alle proprie spalle una lunga storia che si confonde, nella notte dei tempi, con quella stessa della musica. Attualmente, essa intreccia i propri interventi anche con quelli educativi ${ }^{29}$.

La musica addormenta e risveglia, sopisce e lenisce, ma anche eccita ed incita, proprio perché è linguaggio pieno, e coinvolge corpo e spirito.

Da un lato essa si rivolge al cervello arcaico, talamico, disseppellendo gli strati più profondi e primitivi della psiche collegati ai ritmi biologici fondamentali del sonno e della veglia, della quiete e del movimento, dell'aggressione e della pacificazione.

D’altro lato, essa si rivolge pure al cervello più recente, subcorticale e corticale, particolarmente dell'emisfero destro; infine, nelle espressioni della musica dotta, non è possibile escludere anche un coinvolgimento dell'emisfero sinistro, per la sovrapposizione di momenti analitici a quelli sintetici. I suoi effetti d'assieme sono certamente psicosomatici, in maniera indivisibile ${ }^{30}$.

Gli antichi sapevano come la cura del malato non possa limitarsi alle cause strettamente fisiche del suo male, ma debba investirne tutto l'essere.

A parte gli effetti apparenti, il rituale sciamanico otteneva esiti stupefacenti anche sul piano obiettivo migliorando le condizioni generali del paziente, e comunque facendogli superare il vittimismo, la depressione e I'isolamento, ripristinando il circuito sociale e la vita normale ${ }^{31}$.

\footnotetext{
${ }^{28}$ Si può presupporre molto, ma il percorso è forse più lungo. Qualcosa di analogo succede con lo sport; troppi si dichiarano "sportivi» solo perché fruiscono dello spettacolo di massa, senza muovere un dito.

${ }^{29}$ Ciò discende da quanto si diceva all'inizio, e lo conferma.

${ }^{30}$ Non è un caso che la grande medicina antica fosse miticamente collegata alla musica: Iside e Serapide, Apollo e Orfeo suonano e guariscono insieme. Dall'egizio Imhotep ad Esculapio, grande è la considerazione per gli effetti terapeutici dell'arte dei suoni.

${ }^{31}$ Ovviamente, ove esistano guasti anatomopatologici, o cause virali o microbiche, la musica può da sola fare ben poco.
} 
Il suo intervento terapeutico si può dispiegare soprattutto in malattie funzionali o in disturbi in cui abbia parte cospicua una concausa psichica. Ma ci si potrebbe chiedere se vi siano malattie che non abbiano, o non producano esse stesse per via, delle concause psichiche, e talvolta pure sociali.

La malattia è un circolo vizioso che coinvolge quasi tutte le risorse e gli equilibri della persona; I'uomo si ammala tutto intero, guarisce tutto intero ${ }^{32}$.

In maniera in un certo senso paradossale, la malattia può essere considerata come il modo in cui un organismo sano cerca di sopravvivere nonostante un'aggressione: senza di essa, non ci sarebbe guarigione.

La guarigione non è infatti altro che la prosecuzione della reazione vitale quando essa riesce ad aver ragione dell'avversario ed a ripristinare gli equilibri originari.

Che la malattia fosse una specie di lotta contro un avversario, era fantasticamente espresso nella medicina primitiva, che reificava la malattia attribuendola a uno spirito maligno, o a influssi, miasmi, ecc ${ }^{33}$.

L'errore della reificazione c'era anche allora; ma la reificazione vissuta ed empatica aveva effetti innegabili terapeutici complessivi. La reificazione moderna proviene invece dal pensiero razionalistico, che analizza e divide.

Essa ha avuto indubbiamente il merito di aiutare ad isolare le cause specifiche di ogni morbo, classificato in maniera categoriale; sopra tale base è stato edificato il grandioso edificio della diagnosi differenziale, fondata su una semeiotica obiettiva, e della terapia mirata, che ad ogni costellazione morbosa fa corrispondere certi mezzi di intervento.

Oggi non ne potremmo più fare a meno, e sarebbe assurda ogni nostalgia per la medicina antica. C'è comunque da specificare che la musica, protagonista nella medicina antica, ha continuato ad esserlo per secoli nei casi che sembravano sfuggire alla medicina classica, imponendosi a quest'ultima come una presenza di tutto rispetto.

Basta ricordare che le tarantelle hanno avuto origine dalla cura del tarantolismo, vero o immaginario, come il coribantismo ${ }^{34}$.

\footnotetext{
${ }^{32}$ Per inciso, non esiste neppure la malattia come tale, come una entità nosologica astratta, bensì esiste il malato nella sua concretezza individuale, la cui reazione vitale ad una sorta di aggressione costituisce ciò che impropriamente viene reificato come malattia.

${ }^{33}$ La cura si presentava quindi come un rito inteso ad esorcizzare la possessione: e vi avevano un ruolo il guaritore, il paziente, i suoi familiari, talvolta anche l'ambiente, con dispiegamento di gesti rituali, parole, danze, musiche appropriate.
} 
La musica, adeguata alle condizioni del paziente e protratta per diversi giorni, sembrava innescare la risposta di guarigione ${ }^{35}$.

La musica agisce rasserenando, ma soprattutto salda i contatti assenti, quale ponte di passaggio fra il malato (quasi fortezza chiusa) e l'ambiente circostante, favorendo il rientro in attività di gruppo e socializzanti.

La musica ha come proprio componente essenziale il ritmo: e da ritmi ciclici è governata quasi ogni nostra attività fisiologica ${ }^{36}$.

La musica può produrre effetti misurabili in termini di biofeedback sulla regolazione di alcuni ritmi vitali. Può stabilire le condizioni adatte alla quiete e alla meditazione, oppure trascinare in una esaltazione mistica o guerriera.

Ci sono musiche che conducono quasi senza volerlo a battere il tempo o a muovere parti del corpo, e musiche che dapprima accompagnano e poi inducono stati d'animo ${ }^{37}$. Talune di queste musiche hanno un'antichità plurisecolare, ed alcune sono probabilmente addirittura millenarie.

Quando si accetta la sfida, ecco che si scioglie o si scatena la danza. E allora il coinvolgimento è completo. Non c'è movimento che non sia perfettamente sincronizzato e sincronizzante, fino alla più impercettibile vibrazione. Tensione e rilassamento si alternano, gesto e torsione del busto, delle braccia, delle mani, delle dita, delle gambe sono accordati in una espressione mirabile, totale.

È prodigioso come questa armonia, vissuta dall'interno, venga trovata spontaneamente.

Una danza studiata e voluta, come la danza classica, richiede anni di dura disciplina, in cui il corpo viene piegato ad una docilità assoluta. Ma quando giunge il momento magico della performance, l'equilibrio di tutti i momenti è trovato in un unico atto di grandiosa spontaneità creativa, che unifica e salda tutto.

${ }^{34}$ Ed è probabile che con esso venissero confuse anche forme di irrequietezza motoria, di corea o ballo di San Vito, di movimenti spastici e simili.

35 Non diversamente, sono state curate con la musica le manifestazioni di ipocondria, isterismo, malinconia, depressione profonda, anoressia, tendenza al suicidio; in una parola, malattie che potremmo dire prodotte da interruzione dei processi comunicativi. Perfino l'autismo e la schizofrenia, che hanno cause più gravi e profonde, paiono aver tratto beneficio da questo trattamento.

${ }^{36}$ Il battito cardiaco, il respiro, le onde elettriche cerebrali sono solo degli esempi.

${ }^{37}$ Si pensi alle musiche arabe basate su nenie ripetute all'infinito con lievi variazioni, quasi a volerne approfondire ed estrarre l'ultimo senso: all'improvviso la nenia è come lacerata da un'altissima nota, o da un grido, che emerge come una scimitarra lucente. $O$ si pensi alle musiche indiane con i loro strumenti incantatori sollecitati da un tocco delicato. $\mathrm{O}$ a quelle cinesi e giapponesi, che alternano a nenie carezzevoli un fresco tintinnare di rugiada e di perle preziose. 
In casi più semplici, ma non meno pieni di fascino, gli stessi equilibri fisici conducono a trovare le figure più adatte, come nel pattinaggio artistico.

In conclusione: è inconcepibile e impensabile un'educazione senza spirito musicale, inteso quale disposizione ad accogliere e ad esprimere in armonia ben temperata atti e pensieri.

\section{BIBLIOGRAFIA}

COLARIZI, G., 1994, II ritmo nella poesia nella musica nella danza, Edizioni Scientifiche Italiane, Napoli.

DAHLHAUS, C., 2008, L'estetica della musica, Casa editrice Astrolabio, Roma.

DI BENEDETTO, A., 2008, All'origine fu la vibrazione, Nexus edizioni, Padova.

DONÀ, M., 2007, Filosofia della musica, Bompiani, Milano.

HALE, S. E., 2009, Spazio sacro suono sacro. I misteri acustici dei luoghi sacri, Edizioni Mediterranee, Roma.

HARNONCOURT, N., 2008, Glazba kao govor zvuka. Putovi za novo razumijevanje glazbe, Algoritam izdanje, Zagreb.

INNAYAT KHAN, H., 2010, II misticismo del suono, Edizioni Mediterranee, Roma.

LAENG, M., 1990, Movimento gioco e fantasia, Giunti Lisciani, Teramo.

MIQUEL, R., 2002, L'universo delle forme sonore. L'azione e gli effetti del suono sull'uomo, Hermes, Roma.

PLUTARCO, 2008, La musica, Rizzoli, Milano

ROTH, G., 2009, Ispleši svoje molitve. Pokret kao duhovna praksa, Planetopija izdanje, Zagreb.

SACKS, O., 2012, Musicofilia. Racconti sulla musica e il cervello, Adelphi, Milano.

SCHNEIDER, M., 2007, I/ significato della musica, SE, Milano.

TULKU, C., 1998, // signore della danza, Ubaldini Editore, Roma 


\section{GLAZBA I MUZIKOTERAPIJA}

Zašto ljudi vole glazbu? Postoje mnogi odgovori, no nijedan sasvim zadovoljavajući. Međutim, nitko neće prigovoriti ako se odgovori "jer čini dobro" uključujući pritom sve biološke, psihološke, sociološke, estetske i etičke implikacije. U tome smislu, odnosi između općega obrazovanja, glazbenoga obrazovanja i muzikoterapije jako su bliski. Ovim izlaganjem namjeravamo skrenuti pozornost na njih pokazujući kako (njezina) terapijska funkcija nije samo jedno sporedno pitanje ili dopunsko obrazovanju, već više od toga. Zbog toga je nezamislivo da se danas kao nekada razmišlja na potpuno obrazovanje bez glazbe na počasnome mjestu. Štoviše, odgoj je nezamisliv bez glazbenoga duha: kao spremnost prihvaćanja i izražavanja u primjerenom skladu djela i misli.

Ključne riječi: glazba, muzikoterapija, "primijenjena glazba", ritam, sklad, melodija, tonalitet

\section{MUSIC AND MUSICTHERAPY}

Why do people like music? There are several possible answers, but none fully satisfactory. However, nobody will have anything against the answer "because it is good", including all the biological, psychological, sociological, esthetical and ethical implications. In this case, the relationships between general education, musical education and music therapy are even stronger. It is our intent to recall them in order to prove that the therapeutic function is not only marginal or optional in education, but it is much more relevant. It is therefore inconceivable to think of an education without finding a reference with music. Moreover, an education without musical spirit is unthinkable because only in this way it is possible to accept and express actions and thoughts in harmony.

Key words: music, music therapy, "applied" music, rhythm, harmony, melody, tonality 